\title{
Labyrinthe
}

40 | 2013

Comme les abeilles

\section{La pollinisation humaine à l'ère numérique}

\section{Yann Moulier Boutang}

\section{OpenEdition}

\section{Journals}

Édition électronique

URL : http://journals.openedition.org/labyrinthe/4329

DOI : $10.4000 /$ labyrinthe.4329

ISSN : 1950-6031

Éditeur

Hermann

Édition imprimée

Date de publication : 1 mars 2013

Pagination : 125-128

ISBN : 9782705688400

Référence électronique

Yann Moulier Boutang, "La pollinisation humaine à l'ère numérique », Labyrinthe [En ligne], 40 | 2013,

mis en ligne le 01 mars 2015, consulté le 01 mai 2019. URL : http://journals.openedition.org/

labyrinthe/4329; DOI : 10.4000/labyrinthe.4329 


\title{
La pollinisation humaine à l'ère numérique
}

\author{
Yann Moulier BouTANG
}

Adoptons un point de vue strictement évolutionniste, et raisonnons en termes d'intelligence, c'est-à-dire de la faculté de donner une réponse non préprogrammée à une question qui n'a pas été posée avant. Sous cet angle, les notions de connaissance et d'innovation prennent une importance considérable. C'est dans ce cadre que peut être resitué le paradigme de la pollinisation des abeilles. J'en ai fait un paradigme et ça a pris comme une traînée de poudre. J'en ai parlé au début de 2005 et c'est devenu maintenant un lieu commun. Il y a un atelier qui vient de se dérouler à l'Institut Inspire à Porquerolles, au cours d'une université d'été, sur l'économie de pollinisation; il y a d'ailleurs un tag que vous pouvez suivre sur le blog du Monde.

Quelles leçons tirer de la pollinisation ? C'est la modernité extrême de ce mode de fonctionnement vieux comme Hérode, que l'on redécouvre à l'ère numérique dans le cadre d'une réévaluation radicale de l'importance de la sphère non directement marchande - et non marchande tout court. Il peut s'agir d'externalités, lorsque quelque chose a un coût public mais n'est pas une marchandise, donc n'a pas véritablement de prix. (Et s'il y a un prix, il est en fait totalement artificiel.) Mais il faut aussi envisager le non-marchandisable tout court: c'est le cas par exemple de la biosphère, qui n'est pas marchandisable puisqu'aucun coût alternatif n'est calculable. Tant qu'on n'a pas le coût de déménagement de l'humanité ou d'une partie de celle-ci vers une exoplanète, dans un système où l'on pourra vivre, on ne peut pas attribuer un prix à la biosphère. Comme il n'y a pas de «plan B de la biosphère », elle est sans prix. Et quand on dit que quelque chose est sans prix, cela signifie que ce n'est surtout pas le moment de mettre un prix dessus : c'est sans prix pour nous, donc c'est un inconditionnel. Or la pollinisation rentre absolument dans ce cadre puisqu'on est incapable de se substituer artificiellement au travail des abeilles. On a tenté de le faire pour la vanille: il faut polliniser chaque pied de vanille directement et manuellement, c'est infernal. Imaginez si on devait le faire pour la nature, cela n'aurait aucun sens. 


\section{Labyrinthe, $n^{\circ} 40$}

Aux États-Unis, parce qu'on a besoin d'une pollinisation massive, on se met à faire un élevage d'abeilles industrielles. Le Département fédéral de l'agriculture promène des essaims d'abeilles dont les apiculteurs américains louent les services (à titre d'exemple, la pollinisation représente $30 \%$ des coûts de production du colza). Le problème est que ces abeilles-là sont beaucoup moins protégées sur le plan immunitaire. L'apiculteur qui loue leur service, lui, se moque éperdument de leur santé. Du moment qu'elles peuvent polliniser, peu importe ce qui leur arrive pendant le reste de la saison.

Parmi les externalités non encore marchandisées, il y a donc celles sur lesquelles on peut mettre un prix, et les externalités d'un deuxième type, sur lesquelles on ne peut pas mettre un prix. Par exemple la confiance, l'amour. Lorsqu'on commence à mettre un prix sur l'amour, cela devient ou la prostitution ou bien une procédure d'instance de divorce, où l'on dit « je t'ai rendu un service », «j'ai apporté une pile d'assiettes ». Mais en général, il y a des choses qu'on ne met pas en forme, qu'on ne codifie pas sous peine d'abolir complètement la confiance. Je les appelle des « immatériels II ». Et c'est notamment le cas de la coopération, à la différence de la coordination. On ne peut pas enjoindre aux gens de coopérer. Et c'est à mes yeux le ressort fondamental.

Léquivalent humain de la pollinisation, ce sont pour moi tous les comportements sociaux : la sociabilité originelle, l'empathie, la collaboration, la sympathie, la coopération, le jeu, l'intelligence. Il y a bien sûr une différence importante : la pollinisation des abeilles est inconsciente, elle déborde l'intentionnalité, la conscience, la finalité. Mais il y a beaucoup de choses dans les comportements humains qui sont de cet ordre. Par exemple les effets d'externalités positifs de la conversation, de la culture. Quel est l'effet d'un livre ? La valeur d'un livre, comme l'avait très bien dit Gabriel Tarde, ne vient pas du travail nécessaire pour fabriquer le papier, pour payer ceux qui fabriquent le livre. Ce n'est pas non plus le temps de travail mis à l'écrire, qui n'a aucune signification. Vous pouvez donner à un artiste de quoi subvenir à ses besoins, lui donner « une ruche » en quelque sorte, mais vous n'allez pas déterminer le temps de travail comme vous allez déterminer celui de la production d'une chaise. La valeur d'un livre, selon Tarde, tient dans les effets qu'il produit sur ceux qui l'ont lu. On travaille pour le langage, on sait que ce langage va circuler, on sait qu'il y a toujours des choses qui finissent par trouver leur destinataire, comme la bouteille à la mer. Mais dire qu'on a des intentions conscientes 


\section{La pollinisation humaine à l'ère numérique}

et qu'on va faire ceci ou cela, c'est le baratin du cirque « marchand », celui qu'on organise avec des vedettes qui vous disent « voilà, j'ai pensé faire ci, faire ça et j'ai obtenu ce résultat ». Tout le monde le sait, on ne fabrique pas un best-seller. Il n'y a pas de recette du succès.

Comment arrive-t-on à capter les immatériels II ? C'est justement l'enjeu du passage du web 1.0 - vitrine ou vous avez des données accessibles - au web 2.0. Avec ce dernier, vous utilisez un moteur de recherche pour entrer vos propres requêtes, vous utilisez des plateformes qui ne vous coûtent rien et, pendant que vous faites ça, le dispositif informatique enregistre tout ce que vous faites. Vous donnez des informations qui vous concernent, vous communiquez des informations personnelles, ce qui est le propre des réseaux sociaux ou de Google... Et ces informations, que sont-elles finalement? Elles sont la trace de votre propre pollinisation et de la pollinisation collective. Autrement dit, la pollinisation collective se trouve tracée par l'informatique et l'exploitation de ces données de pollinisation permet ce que j'appelle le capitalisme cognitif, le fait de pouvoir capter une partie de la valeur de cette pollinisation. Même si seulement $10 \%$ de cette pollinisation sont captés, cette traçabilité numérique offre des possibilités marchandes incroyables, ce qui pose évidemment de grands problèmes. La fortune d'Apple, aujourd'hui, c'est iTunes. Et iTunes, c'est un véritable écosystème. Aujourd'hui, il y a une concurrence féroce entre ces écosystèmes qui utilisent les données mises sur le web. La prochaine étape, c'est le cloud, le nuage.

Ainsi il va devenir possible de capter des immatériels II comme le care, la compétence, la confiance, la collaboration, le talent, la tolérance, la technologie, à travers des plateformes collaboratives, des moteurs de recherche, des «nuages ». Ce sont de nouveaux modèles économiques marchands, directs ou indirects. Ils soulignent la puissance de l'intelligence collective, et ses implications organisationnelles dans la société et dans l'entreprise. La crise d'aujourd'hui tient à cela: dans une économie de la pollinisation, le fonctionnement ne se fait pas par pyramide hiérarchique, mais par cercles. En étudiant les abeilles, Henry Duchemin parle de cercles semblables à ceux des anneaux olympiques, des cercles qui se nouent entre eux, et permettent de changer d'attribution. Un tel fonctionnement vient complètement déstabiliser l'organisation industrielle antérieure, où les taux de profit des Google et autres sèment le plus grand trouble.

Prenons un exemple, qui montre à quel point toute l'économie matérielle est complètement infusée par l'économie de l'information. Tout 


\section{Labyrinthe, $n^{\circ} 40$}

le monde a parlé de la réussite du modèle BMW. Mais savez-vous quel est le plus gros sous-traitant de BMW en valeur ? C'est tout simplement Dassault Systèmes. Les nouvelles voitures sont en effet construites autour d'un système de captage d'information auprès du pilote et des passagers. Ce système relève la pression artérielle du conducteur, ses mouvements cardiaques, ses tics de langage, etc. Les bases de données ainsi constituées, comme Google - car on peut dire que BMW se « googlise » sont en fait la vraie source de profit de l'entreprise, qui, à la limite, cesse de s'intéresser au profit qu'elle fait sur la tôle qu'elle fabrique. Elle peut presque mettre à disposition une voiture quasi gratuite: ce qui compte, c'est le conducteur et la captation de ses données personnelles, qu'on peut ensuite revendre aux industries pharmaceutiques ou autres. Les systèmes sophistiqués qui gouvernent ces systèmes d'informations ne sont pas comme ceux qui gouvernent la mécanique, mais ils s'appuient sur l'interrelation conducteurs-machines-environnement. On peut prendre encore un exemple, celui d'un très grand fabricant français de plexiglass pour pare-brise : ce fabricant travaille actuellement sur un pare-brise qui va pouvoir projeter à l'avant de la voiture un hologramme, avec une vue du paysage et, comme dans un roman d'anticipation, des vues latérales, des vues arrières, des données d'informations, pour aider le conducteur. La vitre projette un système d'information à partir de capteurs. On est face à une transformation complète de l'économie traditionnelle, qui repose sur des biens matériels.

Tous ces systèmes de pollinisation humaine soulèvent, comme pour les réseaux sociaux, de nombreuses questions, à commencer par celle de la protection de la vie personnelle. Où finissent toutes ces données ? Si le cloud disparaît, que se passe-t-il ? Aujourd'hui, la Commission informatique et libertés, présente dans 29 pays de l'Union européenne, se préoccupe vivement de la durée pendant laquelle les Google et autres peuvent détenir vos données personnelles. Il y a là des régulations fondamentales à prévoir. Tout cela pour dire que la pollinisation humaine est possible avec des conditions, mais que la prédation de cette pollinisation existe aussi. Tout comme il existe des prédateurs des abeilles et de leur activité pollinisatrice. 